\title{
Research on Contribution Rate of Independent Innovation to Economic Growth: Evidence from Changshou District of Chongqing City in China
}

\author{
Xing Wang $\mathbb{D}^{1}$ and Yuhong Wang ${ }^{2}$ \\ ${ }^{1}$ Business School, Hohai University, Nanjing, Jiangsu Province 210098, China \\ ${ }^{2}$ School of Business, Jiangnan University, Wuxi, Jiangsu Province 214122, China \\ Correspondence should be addressed to Xing Wang; wangx7411@163.com
}

Received 12 August 2021; Revised 4 September 2021; Accepted 14 September 2021; Published 31 October 2021

Academic Editor: Bin Liu

Copyright (C) 2021 Xing Wang and Yuhong Wang. This is an open access article distributed under the Creative Commons Attribution License, which permits unrestricted use, distribution, and reproduction in any medium, provided the original work is properly cited.

Using the data of Chongqing Statistical Yearbooks from 2015 to 2020 and based on the Cobb-Douglas production function of Solow residual method, this paper constructs a functional relationship model among GDP, employment number, fixed asset investment, and economic growth. The contribution rate of independent innovation in Changshou District of Chongqing City is improved by using polynomial distributed lag model. Besides, the contribution rate of independent innovation in Shapingba District, Yuzhong District, Jiangbei District, and Beibei District of Chongqing City is compared with that in Changshou District. The research shows that, overall, independent innovation contributes a lot to the economic growth of Changshou District, but its contribution rate is in a slowly declining state. The government needs to take active measures to improve the level of independent innovation and promote the regional economic growth.

\section{Introduction}

Independent innovation is the process of realizing the value of new products through the unique core technology with independent intellectual property rights [1]. It mainly includes three innovation modes: original innovation, integrated innovation, and introduction, digestion, absorption, and reinnovation. Among them, the introduction, digestion, absorption, and reinnovation belong to the primary stage, the integrated innovation belongs to the intermediate stage, and the original innovation belongs to the advanced stage.

In recent years, there have been many empirical studies on independent innovation and economic growth, which confirmed the positive correlation between independent innovation and economic growth [2]. LeBel [3] derived a measure of innovation and tested the extent to which institutional policy choices enhance or delay its diffusion. Hasan and Tucci [4] empirically investigated the importance of both the quantity and quality of innovation on economic growth, controlling for past measures of inventive inputs. Asia-Pacific's fast-developing countries and regions-South Korea, Taiwan Province of China, Singapore, Malaysia, and Thailand-demonstrate the most successful economic development models [5].

By empirical test of vector error correction estimates model, Li and Pan [6] confirmed that there is a long-term stable equilibrium relationship between independent innovation and industrial structure. Wang et al. [7] showed that there was a stable equilibrium relationship between independent innovation and economic growth through the empirical research of provincial panels. From the perspective of technological progress, Zhou et al. [8] put forward a method to measure the transformation of economic growth forces based on theoretical analysis results and pointed out that China's independent innovation showed a good development trend and was expected to become a new source of growth in the future. Zhu and Qi [9] used principal component analysis to consider three national independent 
innovation demonstration zones such as Zhongguancun in Beijing and confirmed that the regional strategy of independent innovation has a significant direct driving effect on economic growth.

Li and Solaymani [10] empirically identify whether technological innovation, sectoral output, and exports growth have had a real impact on industry and agriculture in Malaysia using econometric methods. Ahmad et al. [11] modelled the dynamic linkages between ecoinnovation, urbanization, economic growth, and ecological footprints for G7 countries. Li and Wei [12] demonstrated that carbon emissions will inhibit the positive effects of financial development and innovation on economic growth. Malanski and Póvoa [13] showed that economic freedom works as a moderator in the relationship between corruption and economic growth. Besides, green technology innovation partly mediates the promotion effect of environmental information disclosure on economic development and the inhibition effect of environmental information disclosure on haze pollution under the static situation [14].

Batabyal and Beladi [15] analyzed the impact that stochastically occurring innovations have on Schumpeterian economic growth in a region that is creative in the sense of Richard Florida. Pradhan et al. [16] employed panel unit root and panel cointegration tests to determine the interactions between innovation, financial development, and economic growth in 49 European countries. Maradana et al. [17] used Granger causality approach to research innovation and economic growth in European Economic Area countries. Bucci et al. [18] adopted Hidden Markov Model to investigate the relationship between economic growth and innovation complexity. Shao et al. [19] used a vector error correction model to explore the short-term and long-term relationship between marine economic growth, technological innovation, and industrial upgrading in China. Vural [20] investigated GDP per capita, carbon dioxide emissions per capita, technological innovation, and trade as the determinants of renewable energy production for the selected Latin American countries.

Xiong et al. [21] explored the relationship between R\&D investment and economic growth in China, using a newly collected panel data set. Wang et al. [22] proposed a comprehensive analytical framework to evaluate the efficiency and productivity of green technology innovation. Jian et al. [23] examined the effects of entrepreneurship on economic growth in the context of China's transformation from a centrally planned to a market-oriented economy. Zhou et al. [24] presented a model of technological progress and structural change for economic growth, and the empirical result shows that technological progress has an inverted U-shaped effect on China's economic growth. GM $(1,1)$ is used to predict the coupling and coordination relationship of financial development, technological innovation, and economic growth [25].

The motivation of this paper lies in the construction of functional relationship model among GDP, employment number, fixed asset investment, and economic growth based on the Cobb-Douglas production function of Solow residual method. The main contributions of this paper are summarized as follows: (1) Using the data of Chongqing Statistical Yearbooks from 2015 to 2020 and based on the Cobb-Douglas production function of Solow residual method, this paper constructs a functional relationship model among GDP, employment number, fixed asset investment, and economic growth. (2) The contribution rate of independent innovation in Changshou District of Chongqing City is improved by using polynomial distributed lag model. (3) Besides, the contribution rate of independent innovation in Shapingba District, Yuzhong District, Jiangbei District, and Beibei District of Chongqing City is compared with that in Changshou District.

The remainder of this paper is constructed as follows: Section 2 describes the current situation and existing problems of Changshou District. Section 3 investigates the contribution measurement of independent innovation to economic growth. Contribution rate analysis and policy recommendation are provided in Section 4. Section 5 concludes this paper.

\section{Current Situation and Existing Problems}

Changshou District is an important industrial town in Chongqing and even in western China. In 2020, the total industrial output value above designated size was expected to reach 111.27 billion yuan. In recent years, Changshou District has unswervingly taken the road of new industrialization, relying on the high-tech park and other platforms to focus on cluster development, and vigorously promoted the strategic adjustment of industry and layout. It focuses on improving modern manufacturing such as the chemical industry, transportation equipment, electrical machinery and equipment, and nonferrous metal smelting and processing and accelerating the development of strategic emerging industries such as electronic information, equipment manufacturing, and biomedicine. It also takes solid steps on the path of realizing the ideal prospect of the deep integration of traditional and emerging industries and information technology, the mutual promotion of the public economy and the nonpublic economy, and the cluster development of large, small, and micro enterprises.

At present, there are nearly 400 industrial enterprises above scale, 160 enterprises with annual output value over 100 million yuan, and 4 industrial enterprises with annual output value of 10 billion yuan. The output value of hightech industry, China's famous brand products, and the number of famous brand products of Chongqing rank in the front row of the whole city. According to the data from Chongqing Changshou District Bureau of Statistics, industry is the backbone of Changshou District's economy, with the added value of industry accounting for $41.1 \%$ of the total GDP of the district. In the past five years, although the supporting and leading role of the new industries in Changshou District to the new urbanization has been accelerating and increasing and the pace of building the national innovation-oriented urban area is further accelerated, as a whole, there are still some gaps compared with the specific requirements of economic and social development in the "14th Five-Year Plan" period. 
The period of the "14th Five-Year Plan" is the key period for Changshou District to promote urbanization and industrialization. It is necessary to give full play to the leading role of the government, continuing to deepen the reform of science and technology management system, giving play to the basic role of the market in the allocation of innovation resources, forming the main role of enterprises in innovation, and striving to improve the independent innovation ability of Changshou District. In recent years, although through the implementation of the "innovation-driven strategy" Changshou District has further accelerated the pace of building a national innovative urban area, there are still some gaps compared with the national innovative urban areas. This is mainly manifested in the following aspects.

\subsection{Insufficient Investment in Independent Innovation.} The proportion of R\&D funds in GDP of Changshou District in 2020 was decreasing compared with that in 2019, indicating that the investment in independent innovation of Changshou District was insufficient. The new product development expenses of enterprises above designated size account for $4.2 \%$ of the annual sales revenue of new products, which has not reached the average level of Chongqing, and the science and technology investment of the whole region has not increased with the same amplitude as the regional GDP. Because there is lack of independent innovation risk investment mechanism and core technology and international competitiveness of leading enterprises, the enterprise independent innovation investment level is low.

\subsection{Lack of New Growth Points for Independent Innovation} Industries. In Changshou District, the industry scale based on independent innovation is small, the economic aggregate is small, and the innovation benefit is not high. The traditional industries dominated by Changshou District, such as chemical industry, equipment manufacturing, and nonferrous metal smelting, show a general downward trend under the influence of the macro environment, and the industrial economy continues to be depressed. However, emerging industries such as electronic information, high-end equipment manufacturing, and new materials have not yet produced scale effect and failed to form new growth drivers. In the service sector, the commercial economy accounts for a large proportion, and the modern service industry has not yet developed. The insufficient demand of the real economy is transmitted to the consumption sector. The sales of enterprises selling production materials such as aluminum, steel, and stone materials have declined, and the growth rate of the total sales of goods has slowed down.

\subsection{Independent Innovation Resources Are Relatively Scarce.} By December 2020, it was expected there would be 6,322 R\&D personnel in Changshou District, ranking the 7 th in the nine districts 9th District of the whole city, greatly different from Yubei District. The number of enterprises above designated size with $\mathrm{R} \& \mathrm{D}$ activities was 47 , ranking second. There are 7 research institutions with R\&D activities, ranking the $3 \mathrm{rd}$ in the 9 th district of the whole city. The number of institutions of higher learning with R\&D activities is 7, ranking the third in the ninth district of the whole city. Through the comparison, the independent innovation resources of Changshou District are relatively scarce, and there is a big gap with Yubei District. By December 2020, it was expected that there would be only 12 national and 21 municipal innovative and entrepreneurial talents in Changshou District.

2.4. The System of Independent Innovation Needs to Be Improved. Enterprises in Changshou District have not well combined technology introduction with digestion, absorption, and independent innovation, resulting in the weak independent innovation ability of enterprises. Small- and medium-sized enterprises have weak technical strength, most of which are concentrated in universities, scientific research institutions, and large enterprises. The independent innovation system of enterprises as the main body has not been fully established, and the independent innovation ability of enterprises is weak, so it is difficult for them to adapt to the rapid changes of the market; and the cooperation between enterprises, universities, and scientific research institutions is also insufficient, which leads to the ineffective implementation of the industry-university-research method.

Although the Changshou District government has issued a lot of policies in recent years to help enterprises with independent innovation, these enterprises' expectations for development relying on policies are higher than self-innovation development, which makes enterprises think that independent innovation is not as good as policies and their awareness of independent innovation is weak. Although Changshou District has high-tech zones, the proportion of the output value of high-end technology in regional GDP ranks the sixth in the nine districts of the whole city. It shows that the core technology of high-end technology industry is lacking and the benefit level of independent innovation is very low, which restricts the development of independent innovation in Changshou District. The independent innovation system suitable for Changshou District needs to be further strengthened, the benefits of independent innovation need to be further improved, and the independent innovation mechanism closely combining industry, education, and research is not perfect.

\section{Measurement of Contribution of Independent Innovation to Economic Growth}

In order to study the economic contribution rate of independent innovation in Changshou District, the CobbDouglas production function is improved in this paper. Based on the research of Solow's residual method to estimate the contribution rate of technical progress, the polynomial distributed lag model and semiparametric regression model are introduced into Solow's equation to improve the Solow residual method. In this way, it can avoid directly estimating 
the parameters without satisfying the presuppositions. Then, the contribution rates of independent innovation economic growth in the nine districts of the whole city are obtained, respectively, and the comparative analysis is made accordingly.

3.1. Model and Variable Selection. The contribution rate of independent innovation reflects the efficiency of economic growth, so the quality of economic growth can be evaluated by measuring the contribution rate of independent innovation. Here, total factor productivity is the contribution rate of independent innovation. The parameter estimation model is constructed as formula (1), and the contribution rate of independent innovation to output is constructed as formula (2):

$$
\begin{gathered}
Y=A K^{\alpha} L^{\beta}, \quad(\alpha>0, \beta>0), \\
1=\frac{\ln A}{\ln Y}+\frac{\alpha \ln K}{\ln Y}+\frac{\beta \ln L}{\ln Y} .
\end{gathered}
$$

Output variable $Y$ uses the number of regional GDP of each year, labor input variable $L$ uses the number of employees in the whole society, and capital input $K$ is the total amount of fixed asset investment in the whole society. When the model parameters are estimated, collinearity and heteroscedasticity tests are carried out on the data according to the econometrics theory, and appropriate estimation methods are selected.

\subsection{Preliminary Estimation and Data Verification of the} Model. Before conducting the stationarity test, take the logarithmic time sequence chart of the number of people employed, regional GDP, and fixed asset investment. We found that there was a similar trend among the three, with simultaneous increases in most years. To further explain the relationship between variables, OLS estimation of model parameters was performed according to formula (1).

It can be seen from the calculation results (see Table 1) that the overall explanatory power of the model is relatively high. However, the value of $\operatorname{Ln}(L)$ fails to pass the test, and this model cannot be used to explain the contribution of independent innovation in the process of economic operation. Part of the reason may be that there are various collinearity problems among indicators. Therefore, it is necessary to adopt appropriate methods to eliminate heteroscedasticity and multicollinearity to obtain accurate estimation of model parameters and establish an effective explanatory model. In the real economic society, in many cases, the explained variable $Y$ not only is affected by the explanatory variable $X$ at the same time but also obviously depends on the lag value of $X$. Time lag phenomenon is a common phenomenon in economic and social economy. The causes can be roughly summed up in three aspects: First of all, people's ideas and habits are formed over a long period of time, and it often takes some time to adapt to a new economic environment. Secondly, output and input are often out of sync. Thirdly, due to the rigid constraints of the system, the system cannot make a timely response according to the changes of the market.

3.3. Cointegration Test of Data. Tables 2 and 3 reflect the single integration and cointegration results of the data sequence, respectively.

The single integral test shows that the $t$-statistic of Log $(K)$ is -5.4880 , which is greater than the critical value at the $1 \%$ confidence level and less than the critical value at the $5 \%$ confidence level. That is, rejecting the unit root hypothesis at the $5 \%$ level indicates that there is no trend, that is, a stationary process. The same test result also shows that $\log (Y)$ rejects the unit root hypothesis at the $10 \%$ level, which reflects that the fixed asset investment and the regional GDP do not grow in a completely stable way during this decade.

The cointegration results show that the residual sequence test statistic -3.07 is less than the McKinnon's critical value -2.85 at the significance level of 0.01 . It can be considered that the estimated residuals are classified as a stable sequence, and there is a cointegration relationship between the economic growth rate, the growth rate of fixed asset investment, and the growth rate of employed persons in Changshou District. That is to say, there is a long-term equilibrium relationship among the three.

$\log (Y), \log (K)$, and $\log (L)$, respectively, represent the regional GDP, the total social fixed asset investment, and the number of regional employments after logarithmic transformation.

3.4. Contribution Rate Parameter Estimation. Using the polynomial distributed lag model, the traditional Solow residual estimation model is improved to improve the accuracy and reliability of parameter estimation, and the parameter estimation model about the contribution rate of independent innovation in Changshou District is obtained. The estimated results of the model are reflected in Table 4 and Table 5.

According to the calculation results of the model, both the $R$-squared value and the adjusted $R$-squared value are significantly more than 0.99 , and the fitting effect is very good. In view of the small sample size selected in this paper, DW value is not considered to test autocorrelation here; thus a long-term equilibrium model of contribution rate of independent innovation in Changshou District is obtained:

$$
\ln Y=2.0336+1.1419 \ln L+0.7943 \ln K .
$$

\subsection{Econometric Test of the Model. When Solow residual} method is used to estimate the contribution rate of independent innovation, the traditional method is to adopt the assumption of neutral technological progress. That is to say, the elasticity of substitution of capital to labor is 1 , or the return to scale of the production function is constant; that is, $\alpha+\beta=1$. Then, in the actual calculation, whether this hypothesis is met or not is confirmed by econometric test. In econometrics, the method to test the validity of constraint conditions of model coefficients is Wald test. The test results are shown in Table 6. 
TABLE 1: Parameter estimation results of contribution rate model.

\begin{tabular}{|c|c|c|c|c|c|}
\hline Indicator & Coefficient & $t$-Statistic & Prob. & Test in & \\
\hline$C$ & 1.6938 & 0.3897 & 0.7084 & $R$-squared & 0.9815 \\
\hline $\log (K)$ & 1.0582 & 18.2740 & 0.0010 & Durbin-Watson statistic & 2.1160 \\
\hline $\log (L)$ & -0.5227 & -0.4581 & 0.6608 & $F$-statistic & 186.0244 \\
\hline
\end{tabular}

TABLE 2: A single integral test of a data sequence.

\begin{tabular}{lccc}
\hline Variable & ADF test statistic & Confidence coefficient & Critical value \\
\hline $\log (K)$ & -5.4880 & $1 \%$ level & -5.5219 \\
$\log (Y)$ & -3.4648 & $5 \%$ level & -3.3210 \\
$\log (L)$ & -2.6824 & $10 \%$ level & -2.7711 \\
\hline
\end{tabular}

TABLE 3: Cointegration test of data series.

\begin{tabular}{lcc}
\hline & & $t$-Statistic \\
\hline Augmented Dickey-Fuller test statistic & & -3.0728 \\
& $1 \%$ level & -2.8473 \\
Test critical values & $5 \%$ level & -1.9882 \\
& $10 \%$ level & -1.6001 \\
\hline
\end{tabular}

TABle 4: Contribution rate estimation model.

\begin{tabular}{lcccc}
\hline Variable & Coefficient & Std. error & $t$-Statistic & Prob. \\
\hline$C$ & 2.0336 & 2.2570 & 0.9010 & 0.4627 \\
Log $(K)$ & 0.7943 & 0.1232 & 6.4449 & 0.0232 \\
Log $(L)$ & 1.1419 & 0.4116 & 2.7745 & 0.0091 \\
PDL01 & $1.64 E-07$ & $4.79 E-08$ & 3.4183 & 0.0760 \\
PDL02 & $2.65 E-07$ & $8.32 E-08$ & 3.1806 & 0.0863 \\
PDL03 & -0.0239 & 0.0086 & -2.7952 & 0.1077 \\
\hline
\end{tabular}

TABLE 5: Model test results.

\begin{tabular}{lc}
\hline Indicator & Value \\
\hline$R$-squared & 0.9996 \\
Adjusted $R$-squared & 0.9986 \\
S.E. of regression & 0.0141 \\
Residual sum of squares & $3.96 E-04$ \\
Log likelihood & 28.3070 \\
$F$-statistic & 988.6223 \\
Prob. (F-statistic) & 0.0010 \\
Mean dependent var & 15.3518 \\
S.D. dependent var & 0.3738 \\
Akaike info criterion & -5.5767 \\
Schwarz criterion & -5.5172 \\
Hannan-Quinn criterion & -5.9786 \\
Durbin-Watson statistic & 2.9220 \\
\hline
\end{tabular}

Under the significance of $0.05, F$-test rejects the null hypothesis, and the ending probability is far less than 0.05 . Therefore, the assumption of constant returns to scale is not feasible in this model.

3.6. Measurement of the Contribution Rate of Independent Innovation. In formula (2), $\alpha \ln K / \ln Y$ represents the rate of contribution of fixed asset investment to GDP growth, $\beta \ln L / \ln Y$ represents the contribution of employment to regional GDP growth, $\ln A / \ln Y$ represents the contribution rate of independent innovation to regional GDP growth, and the economic constraint is that they add up to 1 . In equation (2), the parameter estimation value of longevity zone has been obtained. According to Solow estimation equation, the sum of contribution rates is not equal to 1 . This is because it is not regressed by Solow's equation. Even if it is regressed by Solow's equation, it is difficult to add the contribution rate 
under the constraint of 1 due to the prediction error and the lack of sample data. Therefore, this paper calculates the proportion of each contribution rate in the sum of contribution rates and for the measurement of the contribution rate, so that it has more clear economic significance (see Table 7).

In this paper, Shapingba District, Yuzhong District, Jiangbei District, and Beibei District of Chongqing were selected for the comparative study, and the calculation results are shown in Table 8.

Among the above regions, Changshou District has the highest contribution rate of independent innovation, followed by Shapingba District, and Beibei District has the lowest contribution rate. The contribution rate of independent innovation in Changshou District showed a fluctuating trend on the whole and reached the peak of recent years in 2018. Shapingba District was the lowest in 2017, and then it picked up and gradually increased in the later period. However, there was a slight decline in 2020, which should be due to the influence of COVID-19. The contribution rate of independent innovation in Yuzhong District changed steadily but showed a slow declining trend in general, especially in 2020. Jiangbei District and Beibei District have always been below the average level of Chongqing City and reached the highest level in recent years in 2018.

\section{Contribution Rate Analysis and Policy Recommendation}

\subsection{Contribution Rate Analysis}

4.1.1. Independent Innovation Has Made a Large Contribution to Economic Growth. Data show that the contribution of each factor to economic growth in Chongqing can be roughly divided into two stages: before the 2018 Sino-US trade war (2015-2018) and after the 2018 Sino-US trade war (2018-2020). Compared with the previous stage, the contribution rate of independent innovation decreased after the start of the trade war between China and the United States. However, after the government put forward timely measures to stimulate local investment, especially in the manufacturing sector, its contribution rate has picked up in recent years, and its overall trend of change is relatively stable. It should be noted that the contribution rate of independent innovation in Changshou District has shown a slow declining trend in recent years, with an average annual decline of $0.58 \%$. This indicates that industrial upgrading is imminent, and the government needs to strengthen the adjustment of the traditional industrial structure and optimize the industrial layout. At the same time, since the outbreak of the trade war between China and the United States, the government's investment drive and regulation measures have played a certain role, and the level of decline has eased. On the whole, the decline level is relatively small. Changshou District's independent innovation degree is higher than that of the other four districts, and its annual contribution is $11.60 \%$, followed by Shapingba District's $9.97 \%$. In the past two years, the contribution rate of independent innovation in Shapingba District has been continuously improving, showing a tendency to catch up with it. Of course, the innovation contribution rate of Changshou District is far beyond the average level of the whole city, which is inseparable from the unique resource advantage of Changshou District.

4.1.2. Capital Elements Contribute the Most to Economic Growth. According to calculation, the contribution rate of capital factors has reached $65.58 \%$ on average, indicating that the economic growth of this region is mainly driven by capital input, and the contribution rate of capital has always been the largest among the three factors. With the upgrading and adjustment of industrial structure, the new round of development of Changshou District will continue to expand. It is certain that in the future, for a quite long time, the capital factor will still be the main force to maintain economic growth. Changshou District's economic growth is still relatively typical extensive expansion of reproduction, and its economic growth mode has not changed substantially.

4.1.3. The Contribution of Labor Factors to Economic Growth Is Relatively Reasonable. The annual contribution rate of labor factors in Changshou District is $22.86 \%$, showing a decreasing trend year by year. On the one hand, the problem of lack of talents in the region has not been effectively solved. On the other hand, the rise of labor costs has led to the increase of surplus labor force, and the employing units of enterprises cannot recruit suitable staff. From the perspective of Chongqing, the contribution of labor factors in Chongqing is $4.37 \%$, which also shows a decreasing trend year by year, which reflects that the quality of labor force is generally low. As a result, the government needs to increase the spending on on-the-job training and education for the unemployed. Among the five districts, Yuzhong District has the highest contribution rate of labor factors, which reaches $71.17 \%$ annually, and it has been improved in recent years. Beibei District is the next, which is $38.67 \%$, and Jiangbei District is the least, which is $1.12 \%$. This indicates that the regions with relatively developed service industry in the region have higher contribution of labor factors. Yuzhong District, in particular, is the financial center of Chongqing and its financial service industry is very developed. This also shows that it is very important to accelerate the development of the service industry and optimize the industrial structure.

\subsection{Policy Recommendation}

4.2.1. There Is a Need to Make Great Efforts to Improve Our Capacity for Original Innovation, Integrated Innovation, Introduction, Absorption, and Redigestion of Innovation. We should implement the Outline of the Medium- and Long-Term Plan for Scientific and Technological Development and supporting policies and concentrate our efforts on science and technology projects in advanced manufacturing and IT application, new energy and energy conservation technologies, biotechnology and medicine, and environmental protection and public safety technologies. We should accelerate the development of platforms for scientific and 
TABLE 6: Wald test for estimation model of contribution rate of independent innovation.

\begin{tabular}{lccc}
\hline & \multicolumn{2}{c}{ Wald test. Null hypothesis: $c(2)+c(3)=1$} & $\mathrm{df}$ \\
Test statistic & Value & $(1,2)$ & Probability \\
\hline$F$-statistic & 2387.954 & 1 & 0.0004 \\
Chi-square & 2387.954 & 0.0010 \\
\hline
\end{tabular}

TABle 7: Contribution rate of each factor in Changshou District.

\begin{tabular}{lccc}
\hline Year & Employment & Investment & Independent innovation \\
\hline 2015 & 22.9023 & 65.5405 & 11.5572 \\
2016 & 22.4002 & 66.0843 & 11.5154 \\
2017 & 22.5601 & 66.0545 & 11.3855 \\
2018 & 22.2974 & 66.3869 & 11.3157 \\
2019 & 22.2553 & 66.5418 & 11.2029 \\
2020 & 21.8791 & 66.9685 & 11.1524 \\
\hline
\end{tabular}

Note. Data in the table are compiled according to Chongqing Statistical Yearbook.

TABLE 8: Comparison of independent innovation contribution rate of some districts in Chongqing.

\begin{tabular}{ccccccc}
\hline Year & Changshou District & Shapingba District & Yuzhong District & Jiangbei District & Beibei District & Chongqing City \\
\hline 2015 & 11.5572 & 10.0121 & 8.6129 & 3.5586 & 4.2551 & 7.3207 \\
2016 & 11.5154 & 9.9035 & 8.4781 & 4.8283 & 4.1432 & 7.2353 \\
2017 & 11.3855 & 9.1365 & 8.3677 & 5.1086 & 3.9799 & 6.9122 \\
2018 & 11.3157 & 10.0345 & 8.2919 & 6.8872 & 4.7514 & 7.5821 \\
2019 & 11.2029 & 10.5483 & 8.0310 & 5.7952 & 3.6522 & 7.2601 \\
2020 & 11.1524 & 10.1950 & 7.8270 & 6.5002 & 3.6185 \\
\hline
\end{tabular}

technological innovation, such as knowledge innovation, technological innovation, international scientific and technological cooperation, commercialization and incubation of scientific and technological achievements, and public services. We should give priority to the energy conservation and environmental protection industries, high-end equipment manufacturing, and automobile and motorcycle industries and develop next-generation information technology and biopharmaceutical industries in high-tech zones. We should develop deep processing (including consumer-facing end products) while we vigorously build new materials industry.

We should accelerate the establishment of an independent innovation system with enterprises as the mainstay, the market as the guide, and the integration of enterprises, universities, and research institutes, as well as actively promoting the joint reorganization of applied technology research institutes and manufacturing enterprises. We should give full play to the backbone and leading role of key research institutions, institutions of higher learning, and large enterprises in independent innovation. Experience shows that the supporting output value of heavy industry enterprises of 10 billion yuan is more than 30 billion yuan, which drives hundreds of supporting enterprises. The leading role of leading enterprises should be further strengthened. We should strengthen major technological innovation in key areas, industries, and products, improving the industrial chain and reducing transportation costs. We should guide and encourage enterprises to increase investment in scientific and technological research and development, establishing technology centers and truly becoming the main body of $\mathrm{R} \& \mathrm{D}$ investment, the main body of technological innovation activities, and the main body of the application of innovation results. Rational layout of garden within garden construction increases the application and transformation of new technology.

We should improve the environment for independent innovation. We should reform and improve the system of scientific research and innovation in Changshou District and integrate it into the mechanism for the steady increase of state financial investment in science and technology. We should establish and improve the system of investment attraction, establish an industrial investment attraction group based on the industrial foundation of Changshou District, and strive to build a new type of industrial park with "transformation and upgrading" as the main line. We should strengthen investment attraction in industrial chains, focus on pillar industries, work hard to extend the industrial and product chains, make the industrial chains bigger and longer, and promote the deep industrialization of existing industries. We should encourage the development of venture capital for business ventures, establish a diversified investment and financing system for promoting independent innovation, and promote the application of scientific research results and industrial technology vigorously.

4.2.2. There Is a Need to Promote the Development of Modern Service Industries Vigorously. We will further strengthen policy support for the service industry, including extending the implementation scope of preferential tax policies in the Western regions to service enterprises that meet the 
encouraged scope in the national catalogue of industrial restructuring guidance. By means of government procurement and financial discount interest credit, producer service enterprises are encouraged to engage in trans-regional and trans-industrial mergers and reorganizations with trademarks and patents as links. Guide small- and medium-sized enterprises to flexibly adopt brand franchising, brand leasing, and brand creation in parallel, so that their labor costs, marketing channels, customer resources, and other advantages can be organically combined with well-known brands. Leverage well-known brands to expand their own scale and strength, focusing on supporting leading enterprises with high technical content and added value. By the traditional order management transformation brand management, vigorously implement the brand strategy. Actively cultivate service outsourcing enterprises with competitive advantages, creating a number of services outsourcing industrial bases. We should improve the innovation ability and scientific and technological level of outsourcing enterprises and make the service outsourcing industry a new growth point of service trade.

4.2.3. There Is a Need to Give Full Play to the Economic Leverage of Government Investment in Science and Technology. We should encourage research institutions and enterprises to significantly increase their input in scientific and technological development. According to the industrial and technological development requirements of Changshou District, the government's capital investment will be proportionally injected according to the key points. We should increase investment, stabilize investment in real estate, achieve new growth in industrial investment, further increase investment in infrastructure, and establish special funds for technological development of enterprises. In the light of economic development, we should strive to substantially increase financial input in science and technology and at the same time adjust the investment structure. We will establish and improve a multichannel system of scientific and technological investment through fiscal appropriation guidance, financial loan support, enterprise self-financing as the main channel, social fund-raising as the support, and rational use of foreign capital. The management level of scientific research and the amplification effect of government investment in science and technology will be greatly improved.

\section{Conclusion}

This paper advances beyond existing work to some extent. (1) Using the polynomial distributed lag model, the traditional Solow residual estimation model is improved to improve the accuracy and reliability of parameter estimation. The contribution rate of independent innovation can be reasonably obtained by using the semiparameter estimation model of the step-lag model. (2) The Solow residual method has the advantages of clear connotation and simple and wide application in analyzing the contribution of various elements to economic growth. It avoids the defect that its hypothesis premise is difficult to meet in practice, and it is a practical and feasible method. (3) This paper shows that the assumption of constant returns to scale is not feasible in real economic activities. Besides, we calculated the proportion of each contribution rate in the contribution rate and for the measurement of the contribution rate, so that it has more clear economic significance.

This paper has theoretical and practical significance. (1) For theoretical significance, based on independent innovation theory, this paper provides an improved approach for measuring the contribution rate of independent innovation to economic growth. Based on the Cobb-Douglas production function of Solow residual method, this paper constructs a functional relationship model among GDP, employment number, fixed asset investment, and economic growth. Besides, the contribution rate of independent innovation in Changshou District of Chongqing City is improved by using polynomial distributed lag model.

(2) For practical significance, the results clearly reflect the existing problems of independent innovation in Changshou District. Its contribution rate has been in a state of slow decline, but its independent innovation ability has been increasing year by year. On the one hand, the increase of government investment leads to an increase in the proportion of investment contribution. On the other hand, it seems that, in the process of transformation and upgrading, the funds invested in the field of scientific research cannot be innovated in a timely manner, and there is an "incubation" process.

\section{Data Availability}

The statistical data used to support the findings of this study are available from the corresponding author upon request.

\section{Conflicts of Interest}

The authors declare no conflicts of interest.

\section{References}

[1] J. Schumpeter, The Theory of Economic Development, Harvard University Press, Cambridge, MA, USA, 1932.

[2] D. Wu, Y. Wang, and W. Qian, "Efficiency evaluation and dynamic evolution of China's regional green economy: a method based on the Super-PEBM model and DEA window analysis," Journal of Cleaner Production, vol. 264, Article ID 121630, 2020.

[3] P. LeBel, "The role of creative innovation in economic growth: some international comparisons," Journal of Asian Economics, vol. 19, no. 4, pp. 334-347, 2008.

[4] I. Hasan and C. L. Tucci, "The innovation-economic growth nexus: global evidence," Research Policy, vol. 39, no. 10, pp. 1264-1276, 2010.

[5] E. Litsareva, "Success factors of asia-pacific fast-developing regions' technological innovation development and economic growth," International Journal of Innovation Studies, vol. 1, no. 1, pp. 72-88, 2017.

[6] S. Li and Y. Pan, "The impact of independent innovation on the upgrading of industrial structure: an empirical test based 
on VEC model," Jianghan Tribune, vol. 4, no. 7, pp. 56-61, 2016, in Chinese.

[7] C. Wang, Y. Pan, X. Yu, and J. Liu, "Self-innovation and economic growth of the central region: empirical study based on regional panel data in Anhui," Forum on Science and Technology in China, vol. 4, no. 8, pp. 84-89, 2016, in Chinese.

[8] X. Zhou, J. Wang, and Z. Chen, "The propulsion transforms of China's economic growth: based on empirical analysis of technological progress," Inquiry into Economic Issues, vol. 4, no. 6, pp. 1-10, 2018, in Chinese.

[9] Y. Zhu and J. Qi, "Regional development strategy for independent innovation and its driving effect on economic growth," The Theory and Practice of Finance and Economics, vol. 38, no. 5, pp. 104-109, 2017, in Chinese.

[10] Y. Li and S. Solaymani, "Energy consumption, technology innovation and economic growth nexuses in Malaysian," Energy, vol. 232, Article ID 121040, 2021.

[11] M. Ahmad, P. Jiang, M. Murshed et al., "Modelling the dynamic linkages between eco-innovation, urbanization, economic growth and ecological footprints for G7 countries: does financial globalization matter?" Sustainable Cities and Society, vol. 70, Article ID 102881, 2021.

[12] G. Li and W. Wei, "Financial development, openness, innovation, carbon emissions, and economic growth in China," Energy Economics, vol. 97, Article ID 105194, 2021.

[13] L. K. Malanski and A. C. S. Póvoa, "Economic growth and corruption in emerging markets: does economic freedom matter?” International Economics, vol. 166, pp. 58-70, 2021.

[14] Y. Feng, X. Wang, and Z. Liang, "How does environmental information disclosure affect economic development and haze pollution in Chinese cities? The mediating role of green technology innovation," The Science of the Total Environment, vol. 775, Article ID 145811, 2021.

[15] A. A. Batabyal and H. Beladi, "The effects of probabilistic innovations on Schumpeterian economic growth in a creative region," Economic Modelling, vol. 53, pp. 224-230, 2016.

[16] R. P. Pradhan, M. B. Arvin, and S. Bahmani, "Are innovation and financial development causative factors in economic growth? Evidence from a panel granger causality test," Technological Forecasting and Social Change, vol. 132, pp. 130-142, 2018.

[17] R. P. Maradana, R. P. Pradhan, S. Dash et al., "Innovation and economic growth in European Economic Area countries: the granger causality approach," IIMB Management Review, vol. 31, no. 3, pp. 268-282, 2019.

[18] A. Bucci, L. Carbonari, P. M. Gil, and G. Trovato, "Economic growth and innovation complexity: an empirical estimation of a Hidden Markov Model," Economic Modelling, vol. 98, pp. 86-99, 2021.

[19] Q. Shao, L. Chen, R. Zhong, and H. Weng, "Marine economic growth, technological innovation, and industrial upgrading: a vector error correction model for China," Ocean \& Coastal Management, vol. 200, Article ID 105481, 2021.

[20] G. Vural, "Analyzing the impacts of economic growth, pollution, technological innovation and trade on renewable energy production in selected Latin American countries," Renewable Energy, vol. 171, pp. 210-216, 2021.

[21] A. Xiong, S. Xia, Z. P. Ye, D. Cao, Y. Jing, and H. Li, "Can innovation really bring economic growth? The role of social filter in China," Structural Change and Economic Dynamics, vol. 53, pp. 50-61, 2020.

[22] Y. Wang, D. Wu, and H. Li, "Efficiency measurement and productivity progress of regional green technology innovation in China: A comprehensive analytical framework," Technology Analysis \& Strategic Management, 2021.

[23] J. Jian, X. Fan, S. Zhao, and D. Zhou, "Business creation, innovation, and economic growth: evidence from China's economic transition, 1978-2017," Economic Modelling, vol. 96, pp. 371-378, 2021.

[24] X. Zhou, Z. Cai, K. H. Tan, L. Zhang, J. Du, and M. Song, "Technological innovation and structural change for economic development in China as an emerging market," Technological Forecasting and Social Change, vol. 167, Article ID 120671, 2021.

[25] R. Wang and J. Tan, "Exploring the coupling and forecasting of financial development, technological innovation, and economic growth," Technological Forecasting and Social Change, vol. 163, Article ID 120466, 2021. 